\title{
Fano-Rashba effect in a quantum wire
}

\author{
David Sánchez ${ }^{1}$ and Llorenç Serra ${ }^{1,2}$ \\ ${ }^{1}$ Departament de Física,Universitat de les Illes Balears, E-07122 Palma de Mallorca, Spain \\ ${ }^{2}$ Institut Mediterrani d'Estudis Avançats IMEDEA (CSIC-UIB), E-07122 Palma de Mallorca, Spain
}

(Received 31 August 2006; published 27 October 2006)

\begin{abstract}
We predict the occurrence of Fano line shapes in a semiconductor quantum wire with local spin-orbit (Rashba) coupling. We show that the Rashba interaction acts in a strictly one-dimensional channel as an attractive impurity, leading to the formation of purely bound states. In a quasi-one-dimensional system these bound states couple to the conduction ones through Rashba intersubband mixing, giving rise to pronounced dips in the linear conductance plateaus. We give exact numerical results and propose an approximate model capturing the main ingredients of the effect.
\end{abstract}

DOI: 10.1103/PhysRevB.74.153313

PACS number(s): 73.63.Nm, 72.25.Dc, 71.70.Ej

In the Fano effect ${ }^{1}$ the interference of a bound state immersed in a continuum with a nonresonant channel gives rise to characteristic asymmetric line shapes. Fano line shapes have been observed in atomic physics, ${ }^{2}$ Raman scattering ${ }^{3}$ and mesoscopic electron transport, ${ }^{4,5}$ just to mention a few. In all these cases, the origin of the binding potential was clear and the spin degree of freedom played a passive role. Then, it is natural to ask whether a scattering potential coupling the spin and orbital coordinates is able to generate Fano line shapes alone. In this paper we show that the answer is yes.

The latter question is not only of academic interest. There is a continuously growing interest in the potential applications of spintronic devices, where the electron spin is the information-carrying basic unit. ${ }^{6}$ Of special relevance is the experimentally demonstrated manipulation of spin-orbit potentials in low-dimensional semiconductor systems via electric gates. ${ }^{7}$ A type of spin-orbit coupling prominent in certain quantum well heterostructures (typically, InAs based systems) is the Rashba interaction. ${ }^{8}$ It arises when the confinement potential which defines the quantum well is a function of the direction perpendicular to the two-dimensional (2D) electron gas (say, the $z$ direction). This implies a structural inversion asymmetry which, importantly, can be tuned with an external gate potential. ${ }^{7}$ To lowest order in the momentum, the Rashba Hamiltonian reads

$$
H_{R}=\frac{1}{2 \hbar}\left(\left\{\alpha, p_{y}\right\} \sigma_{x}-\left\{\alpha, p_{x}\right\} \sigma_{y}\right),
$$

where the Rashba strength $\alpha$ is proportional to the electric field along $z, \vec{p}=\left(p_{x}, p_{y}\right)$ is the $2 \mathrm{D}$ momentum operator, and $\sigma_{i}(i=\{x, y, z\})$ are the Pauli matrices. The anticommutators in Eq. (1) ensure a Hermitian Hamiltonian when $\alpha$ is nonuniform. Attention to semiconductor spintronics has boomed partly due to the Datta-Das proposal ${ }^{9}$ of an all-electrical spin transistor using the precession effect of a Rashba field onto an injected stream of polarized electrons. The idea is based on a quasi-1D ballistic channel attached to ferromagnetic contacts. ${ }^{9}$ Hence, it is of fundamental importance to investigate transport of electrons subject to spin-orbit coupling along quantum wires. ${ }^{10-14}$ Furthermore, Egues et al. suggest ${ }^{15}$ a quantum wire with local Rashba interaction within a beam-splitter configuration as a tool for entanglement detection via shot noise.

Consider the strict 1D limit of a ballistic quantum wire with local Rashba interaction $\alpha(x): \alpha(x)=\alpha_{0}$ (constant) for $0<x<\ell$ and zero elsewhere. Then, the Hamiltonian in the effective mass approximation is $H=p_{x}^{2} / 2 m-\left\{\alpha(x), p_{x}\right\} \sigma_{y} / 2 \hbar$ and the electron wave function may be expanded, $\psi(x)=\psi_{1}(x) \chi_{+}+\psi_{2}(x) \chi_{-}$, in the spinor eigenstates $\chi_{ \pm}$of $\sigma_{y}$. We now apply the following gauge transformation: $\psi_{1,2} \rightarrow \psi_{1,2} \exp \left[ \pm i \int{ }^{x} k_{R}\left(x^{\prime}\right) d x^{\prime}\right]$, where $k_{R}(x)=m \alpha(x) / \hbar^{2}$. As a result, the Schrödinger equation becomes $-\left(\hbar^{2} / 2 m\right) \psi_{1,2}^{\prime \prime}$ $=\left[E+\left(\hbar^{2} k_{R}^{2} / 2 m\right)\right] \psi_{1,2}$-i.e., the textbook problem of a square-well potential of length $\ell$ and depth $-m \alpha_{0}^{2} / \hbar^{2}$. This simple argument shows that a local Rashba interaction in the 1D limit forms bound states for negative energies. This important fact has been, to the best of our knowledge, overlooked in many works, which consider only positive-energy states. Exceptions are Ref. 16, which predicts bound states in a Rashba disk within a 2D electron gas, and Ref. 17, which discusses the energy spectrum of 2D Rashba billiards.

In a quantum wire, an initial 2D gas is further confined in the $y$ direction yielding a quasi-1D system with electron propagation in the longitudinal $(x)$ direction for each transverse mode. Therefore, one would expect that in the presence of a local Rashba field the traveling waves to be coupled to the bound state due to the $\left\{\alpha, p_{y}\right\} \sigma_{x}$ term in Eq. (1). The coupling implies opposite spin directions in adjacent subbands for a parabolic confinement potential. ${ }^{11,12}$ Since we then have nonresonant transmission interfering with a channel that passes through the bound state, a Fano line shape will develop. ${ }^{14}$ A typical dip in the conductance plateau is shown in Fig. 1. We emphasize that the Rashba interaction given by Eq. (1) plays the role of both the attractive potential and the coupling to the continuum states generating Fano resonances. We refer to this as the Fano-Rashba effect. Remarkably, tuning of the Rashba strength would sweep the resonances across the Fermi energy [see Fig. 2(a)], much like a gate voltage does in a quantum dot. Below, we perform exact numerical calculations to elucidate this effect and put forward an approximate model showing that the Rashba resonance we find is of a generalized Fano form. ${ }^{1}$

We consider a ballistic quasi-1D conductor uniform in the $x$ direction, 


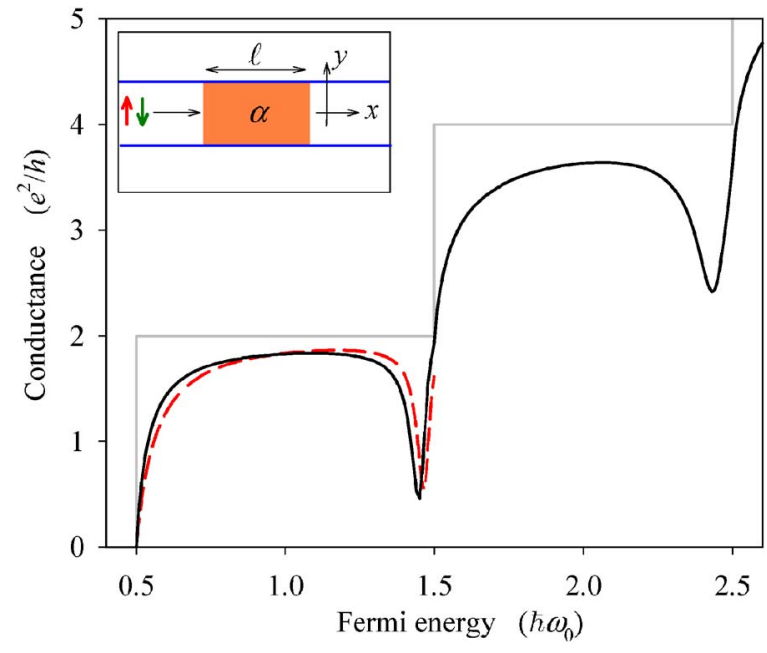

FIG. 1. (Color online) Linear conductance of an electron waveguide with local Rashba interaction (solid line). The solution given by the coupled-channel method is shown with dashed line. We set the Rashba strength $\widetilde{\alpha}=1$ in units of $\hbar \omega_{0} \ell_{0}$ and dot size $\widetilde{\ell}=0.75$ in units of $\ell_{0}$ with $\ell_{0}=\sqrt{\hbar / m \omega_{0}}$ and $\hbar \omega_{0}$ the transversal confinement length and energy scales, respectively. Gray line: the case without Rashba interaction. Inset: sketch of a quasi-1D system with local Rashba interaction.

$$
H=\frac{p_{x}^{2}+p_{y}^{2}}{2 m}+U(y)+H_{R},
$$

where $U(y)$ is the transverse confining potential. It is a good approximation to take $U(y)=m \omega_{0}^{2} y^{2} / 2$, giving transverse modes $\varepsilon_{n}=(n-1 / 2) \hbar \omega_{0}(n=1,2, \ldots)$. The Rashba interaction is localized in a finite region of the wire and tends to zero for $x \rightarrow \pm \infty$. For definiteness, we assume that $\alpha(x)$ is mostly constant and equal to $\alpha_{0}$ in a region of order $\ell$ (a 1D Rashba dot) and quickly vanishes outside with a Fermi function parametrization. ${ }^{18}$ In the results we present below, we take $\hbar \omega_{0}$ and $\ell_{0}=\sqrt{\hbar / m \omega_{0}}$ as the energy and length units and use a normalized Rashba strength, $\widetilde{\alpha}=\alpha_{0} / \hbar \omega_{0} \ell_{0}$, and dot length, $\tilde{\ell}=\ell / \ell_{0}$.

The scattering problem for incoming plane waves is solved exactly only numerically. We use the quantum transmitting boundary method ${ }^{19}$ which yields reliable results for the transmission through nanostructure devices with arbitrary potentials. Figure 1 shows the results for the linear conductance as a function of the Fermi energy, $E$. In the absence of Rashba interaction the conductance is quantized in steps of $2 e^{2} / h$ every time $E$ crosses a transverse-mode energy threshold. We also show the conductance in the case of a strong Rashba interaction $\widetilde{\alpha}=1$ for a small dot. We first note a pronounced dip at energies close to the onset of the adjacent plateau. The plateaus do not reach the conductance quanta because the width of the dip is large. For smaller $\tilde{\alpha}$ the plateaus are not so distorted but the dip is still apparent, even down to zero conductance [see Fig. 2(b)]. We find that the appearance of dips in the conductance plateaus is quite general and the particular dip shape depends on the parameters $\widetilde{\alpha}$ and $\tilde{\ell}$.

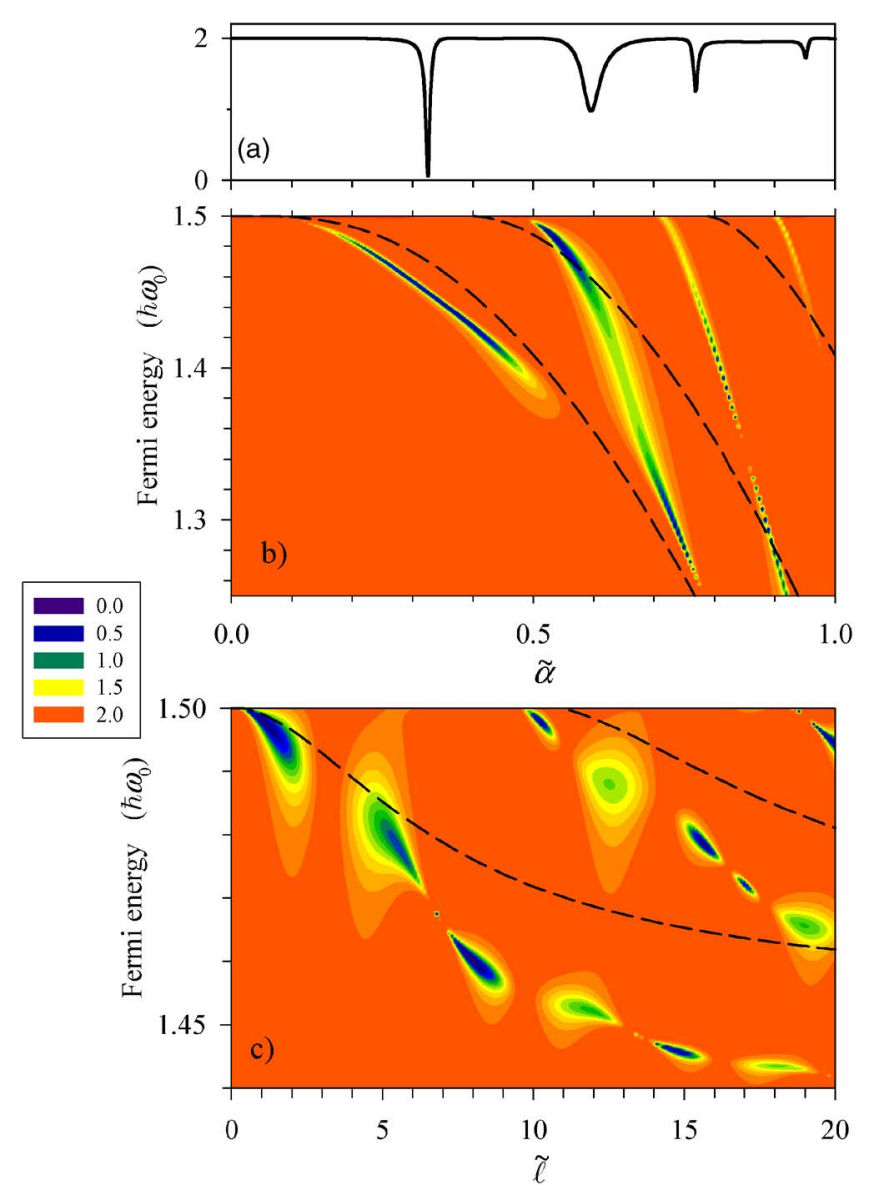

FIG. 2. (Color online) Linear conductance for energies close to the onset of the second plateau $\varepsilon_{2}$. (a) Conductance as a function of the Rashba strength $\tilde{\alpha}$ for a fixed Fermi energy $E=1.45 \hbar \omega_{0}$ $(\tilde{\ell}=8)$. (b) Dependence of the conductance with $\widetilde{\alpha}$ for a fixed dot size $\widetilde{\ell}=8$. (c) Conductance as a function of the dot size for $\widetilde{\alpha}=0.3$. Dashed lines show the $1 \mathrm{D}$ bound state energies relative to $\varepsilon_{2}$.

The occurrence of dips in the conductance curves of quantum point contacts ${ }^{20}$ has been generally associated with the existence of attractive impurities inducing strong backscattering due to resonant reflection. ${ }^{21-23}$ Following Refs. 21 and 22, we expand $\Psi$ in terms of the mode wave functions $\phi_{n}(y)$ :

$$
\Psi(x, y)=\sum_{n s} \psi_{n s}(x) \phi_{n}(y) \chi_{s},
$$

where $\phi_{n}(y)$ describes the motion in the $y$ direction, $\left[-\left(\hbar^{2} / 2 m\right) d^{2} / d y^{2}+U(y)\right] \phi_{n}(y)=\varepsilon_{n} \phi_{n}(y)$. We recall that $\Psi$ is a spinor and $s=\{+,-\}$ labels the spin eigenstates when the spin quantization axis is along $y$. To determine the amplitudes $\psi_{n s}$ we consider an energy lying in the first plateau, $\varepsilon_{1}<E<\varepsilon_{2}$, and assume no significant contributions of states with $n>2$ to the sum in Eq. (3). This two-band model has been used to calculate the energy spectrum of a quantum wire and is a good approach even for large spin-orbit couplings. $^{12}$

Substituting Eq. (3) in the Schrödinger equation and pro- 
jecting onto the mode basis and the spin eigenfunctions we find

$$
\begin{gathered}
{\left[\frac{p_{x}^{2}}{2 m}-\frac{\left\{\alpha, p_{x}\right\}}{2 \hbar}-E+\varepsilon_{1}\right] \psi_{1+}(x)=\frac{i \omega_{p} \alpha}{\hbar} \psi_{2-}(x),} \\
{\left[\frac{p_{x}^{2}}{2 m}+\frac{\left\{\alpha, p_{x}\right\}}{2 \hbar}-E+\varepsilon_{2}\right] \psi_{2-}(x)=-\frac{i \omega_{p}^{*} \alpha}{\hbar} \psi_{1+}(x),}
\end{gathered}
$$

which are coupled-channel (CC) equations. ${ }^{21,22}$ In Eqs. (4) and (5), $\omega_{p}=\int d y \phi_{1}^{*} p_{y} \phi_{2}$. Notably, $\psi_{1+}$ is only coupled to $\psi_{2-}$ because the Rashba intersubband coupling term $\left\{\alpha, p_{y}\right\} \sigma_{x} / 2 \hbar$ connects states with opposite spins. The $\mathrm{CC}$ equations for $\psi_{1-}$ and $\psi_{2+}$ are obtained by the replacements $p_{x} \rightarrow-p_{x}$ and $p_{y} \rightarrow-p_{y}$. Next we introduce the gauge transformation $\psi_{1+, 2-} \rightarrow \psi_{1+, 2-} \exp \left[ \pm i \int^{x} k_{R}\left(x^{\prime}\right) d x^{\prime}\right]$ and obtain

$$
\begin{aligned}
& {\left[\frac{p_{x}^{2}}{2 m}-\frac{\hbar^{2} k_{R}^{2}}{2 m}-\left(E-\varepsilon_{1}\right)\right] \psi_{1+}(x)=V_{12} \psi_{2-}(x),} \\
& {\left[\frac{p_{x}^{2}}{2 m}-\frac{\hbar^{2} k_{R}^{2}}{2 m}-\left(E-\varepsilon_{2}\right)\right] \psi_{2-}(x)=V_{21} \psi_{1+}(x),}
\end{aligned}
$$

where we define

$$
V_{12}=\frac{i \omega_{p} \alpha}{\hbar} \exp \left(2 i \int^{x} k_{R}\left(x^{\prime}\right) d x^{\prime}\right)
$$

and $V_{21}=V_{12}^{*}$. Note that we have transformed the original problem into the CC method of Refs. 21 and 22. However, there are two crucial differences. First, Eqs. (6) and (7) describe spin states and, second, the $V_{i j}$ coupling operators have a nontrivial structure due to the phase factors $\exp \left[2 i \int^{x} k_{R}\left(x^{\prime}\right) d x^{\prime}\right]$.

Equations (6) and (7) cannot, in general, be exactly solved with analytical methods. However, a useful approximation utilizes the ansatz $\psi_{2-}(x)=A \phi_{0}(x),{ }^{22}$ where $A$ is a constant to be found and $\phi_{0}(x)$ corresponds to the wave function of the 1D bound state; i.e., $\phi_{0}(x)$ satisfies $\left(p_{x}^{2} / 2 m-\hbar^{2} k_{R}^{2} / 2 m\right) \phi_{0}(x)=\varepsilon_{0} \phi_{0}(x)$ with $\varepsilon_{0}<0$. Since $E>\varepsilon_{1}$, Eq. (6) describes a $1 \mathrm{D}$ scattering process with a source term given by $V_{12} \psi_{2-}$ and asymptotic wave vector $k=\sqrt{2 m\left(E-\varepsilon_{1}\right) / \hbar^{2}}$. Using the Green function $G$, a formal solution ${ }^{24}$ reads $\left|\psi_{1+}\right\rangle=\left|\varphi_{r}\right\rangle+G V_{12} A\left|\phi_{0}\right\rangle$, where $\left|\varphi_{r}\right\rangle$ is a solution of the homogeneous equation behaving for $x \rightarrow \infty$ as $t e^{i k x}$. As is well known, the Green function for a 1D problem is given in terms of $\varphi_{r}$ and $\varphi_{l}$, the latter going as $t e^{-i k x}$ for $x \rightarrow-\infty$. These just constitute the background or nonresonant channel for direct transmission along the waveguide. Upon substitution in Eq. (7) we find the total transmission

$$
T_{+}(E)=|t|^{2} \frac{\left(E-\varepsilon_{2}-\widetilde{\varepsilon}_{0}+\delta\right)^{2}+(\gamma-\Gamma)^{2}}{\left(E-\varepsilon_{2}-\widetilde{\varepsilon}_{0}\right)^{2}+\Gamma^{2}},
$$

where the parameters are determined from

$$
\left\langle\phi_{0}\left|V_{21} G V_{12}\right| \phi_{0}\right\rangle \equiv \Delta+i \Gamma
$$

$$
\frac{m}{i \hbar^{2} k t}\left\langle\varphi_{l}^{*}\left|V_{12}\right| \phi_{0}\right\rangle\left\langle\phi_{0}\left|V_{21}\right| \varphi_{r}\right\rangle \equiv \delta+i \gamma
$$

These are complicated functions of $E$ but we have checked that their variation is slow around the energy $\varepsilon_{2}-\varepsilon_{0}$. Moreover, the original bound-state energy becomes renormalized to $\widetilde{\varepsilon}_{0}=\varepsilon_{0}+\Delta$, with a shift given by Eq. (10). We stress that Eq. (9) shows resonancelike behavior solely due to the intersubband mixing of the Rashba interaction. The importance of Rashba intersubband mixing has been emphasized in a number of works. ${ }^{11,12,25}$ If one neglects it in Eq. (1), the bound state $\left|\psi_{2-}\right\rangle$ splits off the continuum and does not contribute to the current. Then, one simply has $T_{+}(E)=|t|^{2}$.

The transmission (9) can be cast in the generalized Fano form

$$
T_{+}(E)=|t|^{2} \frac{|\epsilon+q|^{2}}{\epsilon^{2}+1},
$$

where $\epsilon=\left(E-\varepsilon_{2}-\widetilde{\varepsilon}_{0}\right) / \Gamma$ contains the explicit energy dependence and the asymmetry parameter $q=\delta / \Gamma+i(\gamma / \Gamma-1)$ is a complex quantity. As a result, there is no transmission zero unless $\gamma=\Gamma$ for some particular set of parameters (see Fig. $2)$. This is a consequence of the nontrivial form of the coupling operators in Eq. (8) since for real couplings (or couplings satisfying special properties ${ }^{22}$ ) one always finds real $q$ 's. The fact that $q$ develops an imaginary part has been related to the lack of time-reversal symmetry, ${ }^{5,26}$ but since the Rashba interaction is time-reversal invariant, here we have proved that breaking this symmetry is not a necessary condition for complex $q$ 's.

The conductance follows from $\mathcal{G}=\left(T_{+}+T_{-}\right) e^{2} / h$, where the spin-down transmission $T_{-}$is found in a similar way to $T_{+}$solving the CC equations for $\psi_{1-, 2+}$. We find $T_{+}=T_{-}$since the Rashba interaction is not able to produce spin-polarized currents due to time-reversal invariance. ${ }^{27}$ The result for $\mathcal{G}$ is shown in Fig. 1, in rather good agreement with the exact numerical calculation.

The limit of strong spin-orbit coupling is not, however, realistic. For an InAs wire with $\alpha \simeq 10 \mathrm{meV} \mathrm{nm} \mathrm{Refs.} \mathrm{7,} \mathrm{10,}$ and 13 and energy spacing $\hbar \omega_{0} \simeq 1 \mathrm{meV}$, we obtain $\widetilde{\alpha} \simeq 0.2$. For small $\widetilde{\alpha}$ the state is weakly bound and strongly couples to the continuum, breaking the validity of the ansatz for $\left|\psi_{2-}\right\rangle$, and the agreement with the numerics is thus only qualitative. Nevertheless, the resonance line shape is still of the Fano form. In Figs. 2(b) and 2(c) we show an exhaustive study of the dip depth as a function of the Rashba strength and the dot size. We plot at the same time the position of the bound states (dashed lines), which do not match the dip minima due to renormalization. Importantly, we observe dips going all the way down to zero within numerical precision. Increasing $\tilde{\alpha}$ or $\tilde{\ell}$ leads to more bound states. However, the coupling to the continuum decreases when increasing the level binding. Thus, typically, two dips coexist in a given plateau provided they are small. In Fig. 2(a) we show how, for a fixed value of $E$, one can tune the resonances by varying $\widetilde{\alpha}$, which may be regarded as a gate voltage. This is a central prediction of our work, which can be tested experimentally. 


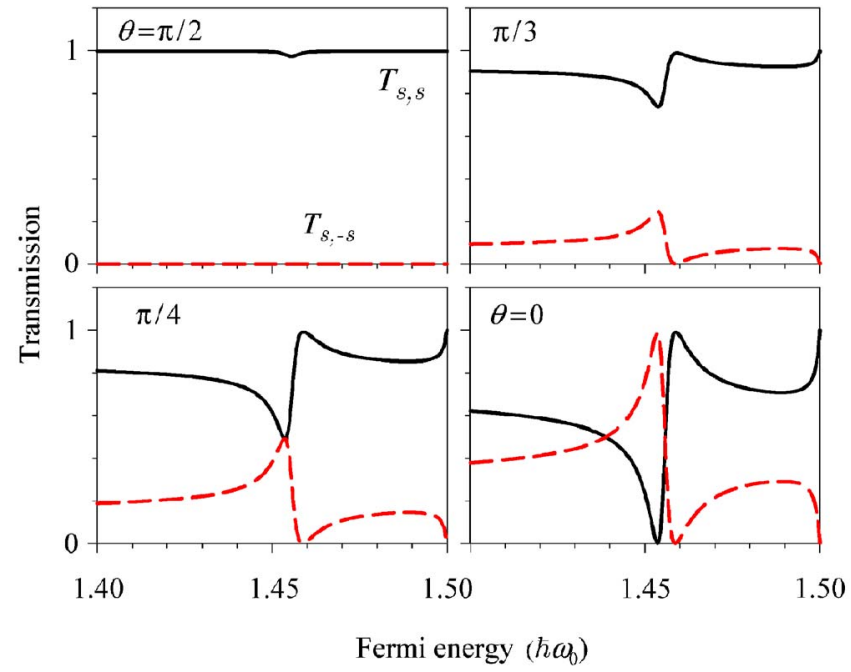

FIG. 3. (Color online) Spin-dependent transmissions for $\widetilde{\alpha}=0.3$ and $\tilde{\ell}=9.5$ when the spin quantization rotates from $y$ $(\theta=\pi / 2)$ to $x(\theta=0)$. The total transmission $T=\Sigma_{s, s^{\prime}} T_{s, s^{\prime}}$ is the same in all cases.

Thus far we have taken the spin quantization axis along $y$, for which both spin channels are equivalent and uncoupledi.e., $T_{+}=T_{-}$and no spin flip. This is no longer true along other orientations and, generally, one must consider the four contributions to the total transmission $T=\Sigma_{s, s^{\prime}} T_{s, s^{\prime}}$, where $s$ denotes the spin state in the left lead (incident) and $s^{\prime}$ that of the right lead (transmitted). Obviously $T$ does not depend on the chosen quantization axis. Besides, due to time-reversal symmetry we always have $T_{++}=T_{--}$and $T_{+-}=T_{-+}$(Ref. 27).
In Fig. 3 we show the spin-resolved transmissions when the spin axis is rotated on the plane from $\theta=\pi / 2$ ( $y$ axis) down to 0 ( $x$ axis). Quite remarkably, $T_{s, s}$ and $T_{s,-s}$ develop conspicuous Fano line shapes with exact transmission zeros for $T_{s,-s}$ when decreasing $\theta$, even for cases in which the total transmission has a vanishingly small dip. Therefore, experiments using ferromagnetic leads with tilted spin orientation would greatly enhance the Fano line shapes. The precise line shapes in Fig. 3 can be explained with the dependences $T_{++} \propto \cos ^{2}(\vartheta / 2)$ and $T_{+-} \propto \sin ^{2}(\vartheta / 2)$, where $\vartheta$ gives the phase difference between the transmission amplitudes with the spins quantized along $y$. Since $\vartheta$ must change rapidly between 0 and $\pi$ in the presence of a bound state, ${ }^{24}$ there must be a sudden variation of the line profile related to the scattering through a resonance.

In conclusion, we have demonstrated that for a ballistic quantum wire with local spin-orbit coupling the conductance shows dips that arise from the interference between bound states formed by the Rashba interaction and the electrons in the conduction channel. The Rashba interaction is also responsible for the coupling between the resonant and nonresonant channels, containing phases that change the position and asymmetry of the resonance in a nontrivial way. For strong enough spin-orbit couplings these properties will be robust against interaction and charging effects, although their detailed investigation requires alternative approaches to the scattering theory used here. We believe that the observation of the Fano-Rashba effect addressed in this work is within the scope of present experimental techniques.

We acknowledge R. López for valuable discussions. This work was supported by Grant No. FIS2005-02796 (MEC) and the "Ramón y Cajal" program.
${ }^{1}$ U. Fano, Phys. Rev. 124, 1866 (1961).

${ }^{2}$ R. K. Adair, C. K. Bokelman, and R. E. Peterson, Phys. Rev. 76, 308 (1949).

${ }^{3}$ F. Cerdeira, T. A. Fjeldly, and M. Cardona, Phys. Rev. B 8, 4734 (1973).

${ }^{4}$ J. Göres, et al., Phys. Rev. B 62, 2188 (2000).

${ }^{5}$ K. Kobayashi, H. Aikawa, S. Katsumoto, and Y. Iye, Phys. Rev. Lett. 88, 256806 (2002).

${ }^{6}$ I. Zutic, J. Fabian, and S. Das Sarma, Rev. Mod. Phys. 76, 323 (2004).

${ }^{7}$ J. Nitta, T. Akazaki, H. Takayanagi, and T. Enoki, Phys. Rev. Lett. 78, 1335 (1997); G. Engels, J. Lange, Th. Schäpers, and H. Lüth, Phys. Rev. B 55, R1958 (1997).

${ }^{8}$ E. I. Rashba, Fiz. Tverd. Tela (Leningrad) 2, 1224 (1960). [Sov. Phys. Solid State 2, 1109 (1960)].

${ }^{9}$ S. Datta and B. Das, Appl. Phys. Lett. 56, 665 (1990).

${ }^{10}$ Y. Sato, S. Gozu, T. Kikutani, and S. Yamada, Physica B 272, 114 (1999).

${ }^{11}$ F. Mireles and G. Kirczenow, Phys. Rev. B 64, 024426 (2001).

${ }^{12}$ M. Governale and U. Zülicke, Phys. Rev. B 66, 073311 (2002).

${ }^{13}$ Th. Schäpers, J. Knobbe, and V. A. Guzenko, Phys. Rev. B 69, 235323 (2004).

${ }^{14}$ L. Zhang, P. Brusheim, and H. Q. Xu, Phys. Rev. B 72, 045347 (2005).

${ }^{15}$ J. C. Egues, G. Burkard, and D. Loss, Phys. Rev. Lett. 89, 176401 (2003;) J. C. Egues, G. Burkard, D. S. Saraga, J. Schli- emann, and D. Loss, Phys. Rev. B 72, 235326 (2005).

${ }^{16}$ M. Valín-Rodríguez, A. Puente, and Ll. Serra, Phys. Rev. B 69, 085306 (2004).

${ }^{17}$ A. Csordás, J. Cserti, A. Pályi, and U. Zülicke, cond-mat/ 0512397 (unpublished). J. Cserti, A. Csordás, and U. Zülicke, Phys. Rev. B 70, 233307 (2004).

${ }^{18}$ We take $\alpha(x)=\alpha_{0}[f(x-\ell / 2)-f(x+\ell / 2)]$, with $f(x)=1 /\left(1+e^{x / \sigma}\right)$. In the numerical simulations we assume a small diffusivity $\sigma$ $=0.2 \ell_{0}$ but the results are not very sensitive to the precise value of $\sigma$ provided $\sigma<\ell$.

${ }^{19}$ C. S. Lent and D. J. Kirkner, J. Appl. Phys. 67, 6353 (1990).

${ }^{20}$ J. Faist, P. Gúeret, and H. Rothuizen, Phys. Rev. B 42, 3217 (1990).

${ }^{21}$ S. A. Gurvitz and Y. B. Levinson, Phys. Rev. B 47, 10578 (1993).

${ }^{22}$ J. U. Nöckel and A. D. Stone, Phys. Rev. B 50, 17415 (1994).

${ }^{23}$ See, however, V. Gudmundsson, C.-S. Tang, and A. Manolescu, Phys. Rev. B 72, 153306 (2005), for conductance dips arising from a repulsive potential in the presence of a magnetic field.

${ }^{24}$ C. J. Joachain, Quantum Collision Theory (North-Holland, Amsterdam, 1975).

${ }^{25}$ Ll. Serra, D. Sánchez, and R. López, Phys. Rev. B 72, 235309 (2005).

${ }^{26}$ A. A. Clerk, X. Waintal, and P. W. Brouwer, Phys. Rev. Lett. 86, 4636 (2001).

${ }^{27}$ L. W. Molenkamp, G. Schmidt, and G. E. W. Bauer, Phys. Rev. B 64, 121202(R) (2001). 